\title{
BOTULISM FROM CHEESE
}

\author{
M A R Y NEVIN \\ From the Division of Laboratories and Research of the New York State Department of \\ Health, Albany
}

On Oct. 19, 1914, a sample of home-made cottage cheese was sent for examination to the Laboratory of the New York State Department of Health, by Dr. Charles G. Duryea, sanitary supervisor. Three persons who had eaten the cheese had died. The clinical data furnished by Dr. Willard Gillette and Dr. Charles G. Duryea suggested different diagnoses, including poliomyelitis, and a definite diagnosis was not madè. Two cases were identical; paralysis of the muscles of deglutition, suffusion of the face, ptosis, total dilatation and failure of pupils to react to light, paralysis of the muscles of the throat with difficult speech were present. There was no loss of consciousness or paralysis of any other part of the body. The third patient was unable to swallow, and in the throat a considerable quantity of mucus accumulated. No eye symptoms were evident, doubtless owing to the fact that it was a milder infection, as the death occurred several days after that of the other two patients.

It is noteworthy that Dr. Duryea, in his search of the literature, recognized that the clinical picture in these patients corresponded to that described by Ostertag, van Ermengem and other investigators of botulism.

Necropsy examination was made and Dr. E. Kellert of the Bender Laboratory, Albany, reported:

Specimen consists of the brain of an adult and a small fragment of spinal cord from the upper cervical region. The specimens are received in a tin pail containing salt solution. The cortical vessels are deeply injected. At the base of the brain the pia appears to be slightly thickened and opaque. No exudate or gross lesion is visible.

Sections from the pons and cord show no cellular infiltration or lesion about the vessels. The white matter appears normal. Many of the ganglion cells are pale-staining, slightly shrunken and have irregular margins. The nuclei are very pale or absent. The Nissl bodies in certain of the cells are pale or absent. In the immediate vicinity of such cells there is an accumulation of numerous granules varying greatly in size and staining deeply with methylene blue and hematoxylin. A few lymphocytes are present in the region of the anterior horns.

Received for publication Nov. 30, 1920. 
Cultures from the ventricles showed a mixed infection in which B. coli predominated. Stained sections from the cord and pons were referred to $\mathrm{Dr}$. Simon Flexner of the Rockefeller Institute, who found no evidence of poliomyelitis. About $30 \mathrm{cc}$ of bloody fluid from the skull were also inoculated by Dr. Flexner into monkeys with negative results.

Bacterial Examination of the Cheese.-When the sample of cheese reached the laboratory, emulsions were made and inoculated into guineapigs and at the same time cultures to isolate the bacteria were made. It was found that the subcutaneous inoculation of $3 \mathrm{cc}$ of an emulsion of the cheese after incubation at $37 \mathrm{C}$. for 48 hours, killed guinea-pigs within 36 hours. Attempts to isolate the bacteria, which might have been the causative agents, were then made. Under aerobic conditions, a staphylococcus, B. coli and B. subtilis were obtained. In anaerobic cultures another organism was found. After heating the emulsion of the cheese at $85 \mathrm{C}$. for 10 minutes, only two organisms were found in anaerobic agar cultures of the material. One of these was B. subtilis found in the aerobic cultures and the other a bacillus, also with spores, but which developed colonies only in anaerobic cultures, which were distinctive.

This organism, somewhat smaller than B. subtilis, was a motile bacillus with rounded ends, and was gram-positive, except in older cultures, when it often decolorized. Oval spores developing early were situated about the end of the middle third. In the adult stage these spores appeared to be terminal. The thermal death point was found to be 80 after one-half hour of exposure and $85 \mathrm{C}$. after 15 minutes of exposure. The organism had no demonstrable capsule in vitro or in vivo.

Gelatin was liquefied slowly and milk coagulated in three days; dextrose, galactose, levulose, lactose, saccharose, maltose, mannit, dextrin, inulin and glycerine were fermented with the production of gas and the odor of butyric acid, which was marked.

According to van Ermengem, ${ }^{1}$ the strain from Ellezelles did not coagulate milk, but von Hibler found that the strain of B. botulinus from Ellezelles, the Damstadt strain and a third isolated from meat often precipitated and peptonized casein, with energetic gas production. The Ellezelles strain that von Hibler worked with had lost its toxicity. Van Ermengem and others observed that the addition of peptone to the medium was necessary for the growth of $\mathrm{B}$. botulinus.

1 Kolle and Wassermann: Handb. d. path. Mikroorg., 1912, 4, p. 920. 
On the contrary, the writer found that potent toxins were obtained in the following medium, made without the addition of peptone:

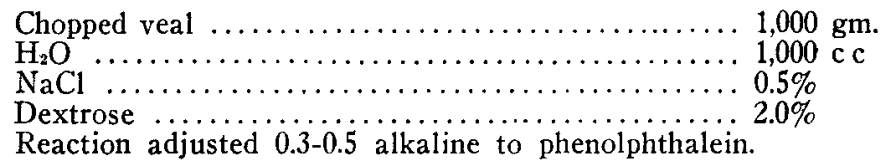

Mice, guinea-pigs and rabbits were killed by the inoculation of cultures of this organism and also by Berkefeld filtrates of the cultures, which were found to be equally toxic.

No special study was made of the conditions under which the more potent toxins were produced. However, contrary to recorded observations of other investitgators, that the bacillus forms toxin and spores only when incubated below $30 \mathrm{C}$, the bacillus not only grew luxuriantly in medium containing dextrose, at $37 \mathrm{C}$. in from 18 to 24 hours and developed spores in 48 hours, but it also produced potent toxins. Guinea-pigs were killed in 4 days with $0.0005 \mathrm{c} c$ of the filtrate of a 72 hour culture.

After inoculation, a period of incubation was followed by loss of accommodation, partial paralysis of the hind legs, dragging of the abdomen; hypersecretion of mucus from the nose and mouth and accelerated respiration were noted, but no fever. No pigs developing these symptoms recovered. At necropsy, a slight congestion was noted at the point of inoculation, and in animals which received cultures the bacillus was found in the edema which was present. Varying degrees of congestion of the lymphatic glands were found and also of the suprarenals, such as is seen after the inoculation with diphtheria toxin. The reaction induced by pure cultures, as compared with those obtained from culture filtrates, differed only at the site of inoculation. In all the animals used, only once was the bacillus found in an organ, namely, in the liver.

In order to obtain the classic picture described by van Ermengem, a kitten weighing $720 \mathrm{gm}$. was inoculated subcutaneously with 5 c.c. of toxin. Within 36 hours, it developed marked symptoms of botulism: hypersecretion from nose and mouth, paresis of the hind legs, mydriasis and intermittent hiccoughs. In 72 hours, the symptoms had developed so far that the animal was unable to move from its side. Partial paralysis developed in the front legs, the pupils were dilated and showed but slight response to light; all food was refused. The weight of the animal dropped from 720 to $565 \mathrm{gm}$., and it was chloro- 
formed on the fifth day. At necropsy, congestion and degenerative changes in the parenchyma of the organs, notably of the liver, were found and retention of the urine and feces.

For the final identification of the organism, cultures of $\mathrm{B}$. botulinus from the Museum of Natural History and from the Medical School of Harvard University, were carried through the different mediums with this organism for the purpose of comparison. The cultural characteristics corresponded in all particulars, but guinea-pigs inoculated with $3 \mathrm{cc}$ of a 48-hour broth culture of both these strains failed to develop any evidence of intoxication.

Although the early investigators thought that botulinus toxin was produced only in the presence of meat protein, it has been found that the poison is produced in the presence of vegetable protein (Dickson ${ }^{2}$ ). In my study, I found that $B$. botulinus grew and produced toxin in ordinary cottage cheese. An emulsion of fresh cottage cheese was sterilized, inoculated with one loopful of a 48-hour broth culture of the organism and incubated at $37 \mathrm{C}$. for 72 hours. A guinea-pig injected with $1 \mathrm{cc}$ of the emulsion died in less than 15 hours. Postmortem examination showed that the veins of the subcutaneous tissues were intensely congested, radiating from the point of inoculation to the axillae and groin. The liver was mottled and the suprarenals hyperemic.

The same technic was followed with cheese not sterilized. The guinea-pig inoculated with $1 \mathrm{cc}$ died in $2 \frac{1}{2}$ days, showing the same picture. For purposes of control, an emulsion of market cheese which had not been sterilized was incubated for 72 hours at $37 \mathrm{C}$. A guineapig inoculated with $1 \mathrm{cc}$ showed no evidence of intoxication.

Further studies of the toxin and its action on the tissues were carried out with a view to studying the development of antitoxin in the animal body. Van Ermengem observed a certain degree of resistance in cats and rabbits so that they withstood more than the lethal dose of toxin for other animals, such as the guinea-pig. But all these animals died with cachectic symptoms, and no marked immunity was induced. Tschitschkine, ${ }^{3}$ by giving small quantities of toxin by mouth, succeeded after great difficulty and the loss of numerous rabbits, in inducing in the rabbit a resistance against a fatal subcutaneous dose. This serum possessed but slight antitoxic value.

2 Jour. Am. Med. Assn., 1915, 65, p. 492.

3 Ann. de 1'Inst. Pasteur, 1905, 19, p. 335. 
Forsman ${ }^{4}$ records the best results in the immunization of small animals by injecting a toxin which was heated to $60 \mathrm{C}$. for 35 minutes. The immunized animals withstood one hundred times the lethal dose. Their blood serum was highly antitoxic.

Larger animals (goats and horses) were first immunized by Kempner, ${ }^{5}$ then by Forssman and Lundström ${ }^{6}$ and more recently by Leuchs. ${ }^{7}$

In my attempts to immunize rabbits with the toxin, 3 animals were lost before any successful results were obtained. The fourth rabbit was inoculated every seventh day and with gradually increasing doses beginning with $0.001 \mathrm{cc}$. The first 3 inoculations were intravenous, the next 8 were intraperitoneal. At this stage the animal was withstanding $0.3 \mathrm{c} \mathrm{c}$ of a toxin the M L D of which for a $300 \mathrm{gm}$. guineapig was 0.009 , and 0.04 of which had killed a rabbit on the fourth day. From this point, a modification of Forssman's method was used. Instead of heating the toxin at $60 \mathrm{C}$. for 35 minutes, it was put in the water bath and the water brought to $60 \mathrm{C}$. After cooling, $1 \mathrm{cc}$ of the toxin was inoculated subcutaneously into the rabbit. The animal withstood an intravenous inoculation of $5 \mathrm{c} c$ of this heated toxin. At its last inoculation it was given subcutaneously $35 \mathrm{c} \mathrm{c}$ of this heated toxin, the minimal lethal dose of which when fresh was $0.01 \mathrm{c} \mathrm{c}$ for a $325 \mathrm{gm}$. guinea-pig. The blood serum of the animal was found to possess antitoxic value, $1 \mathrm{c} c$ protecting a guinea-pig against 100 times the minimal lethal dose of toxin.

Shortly after this paper was read before the American Public Health Association at Rochester in September, 1915, this strain of B. botulinus which I had isolated was sent, on request, to the Department of Agriculture at Washington, and used in the experimental work on forage poisoning by Buckley and Shippen. ${ }^{8}$ Shippen ${ }^{9}$ used this strain exclusively in his work on toxin production, because other cultures obtained from Dickson (originally from Zinsser and the New York Museum of Natural History) produced no toxin.

I have done no work on the typing of this organism, but Burke ${ }^{10}$ and others have found my strain to be of type B, conforming to the strains subsequently isolated in the eastern part of the United States and those obtained from Germany.

4 Centralbl. f. Bakteriol., I, O., 1905., 38, p. 463.

- Ztschr. f. Hyg. u. Infektionskr., 1897, 26, p. 481.

- Ann. d. l'Inst. Pasteur, 1902, 16, p. 294.

7 Ztschr. f. Hyg. u. Infektionskr., 1910, 65, p. 60.

8 Jour. Am. Vet. Med. Assn., 1916, 50, p. 809.

- Arck. Int. Med., 1919, 23, p. 346.

to Jour. Bacteriol., 1919, 4, pp. 541 and 555 . 


\section{SUMMARY AND CONCLUSIONS}

Three fatal cases presented the typical picture of botulism, and B. botulinus was isolated from the cheese eaten by them.

It is evident from these results that meat protein is not necessary to the growth and consequent toxin production by $B$. botulinus, and that cheese may serve as a medium.

There is little significant variation in the cultural characteristics from the type, but contrary to the observations of van Ermengem and others, B. botulinus grows luxuriantly and produces spores and toxin at $37 \mathrm{C}$. The addition of peptone to the medium used is not necessary as in this work potent toxins were produced without the addition of peptone.

A protective serum was produced in rabbits against the homologous toxin. It has been impossible, however, to test its antitoxic value with toxins from heterologous strains of the organism as the strains procured failed to produce toxin.

This appears to be the first time that $\mathrm{B}$. botulinus has been isolated from cheese, that a soluble bacterial toxin has been detected in cheese. and that $\mathrm{B}$. botulinus has been isolated in America. 